\title{
Article \\ Seawater Temperature Prediction Method for Sustainable Marine Aquaculture
}

\author{
Takanobu Otsuka $^{1, *}$, Yuji Kitazawa ${ }^{2}$ and Takayuki Ito ${ }^{1}$ \\ 1 Nagoya Institute of Technology, Japan \\ 2 Nichiyu Giken Kogyo Co. LTD, Japan \\ * Correspondence: otsuka.takanobu@nitech.ac.jp
}

\begin{abstract}
Aquaculture is growing ever more important due to the decrease in natural marine resources and increase in worldwide demand. To avoid losses due to aging and abnormal weather, it is important to predict seawater temperature in order to maintain a more stable supply, particularly for high value added products, such as pearls and scallops. The increase in species extinction is a prominent societal issue. Furthermore, in order to maintain a stable quality of farmed fishery, water temperature should be measured daily and farming methods altered according to seasonal stresses. In this paper, we propose an algorithm to estimate seawater temperature in marine aquaculture by combining seawater temperature data and actual weather data.
\end{abstract}

Keywords: WSN, IoT, seawater temperature prediction, marine aquaculture support

\section{Introduction}

In a discussion of aquaculture, it is important to understand the aquaculture environment. Unlike wild fish and shellfish, fish and shellfish bred in an aquaculture environment are restricted by tanks and rafts and cannot move freely. As a result, fish and shellfish maintained in restricted fishing grounds are at risk of annihilation when fluctuations in red tides and seawater temperatures occur. As a representative example, in 1996, Mie Prefecture's Shingo Bay, a typical pearl culture aquaculture pond, suffered a massive die-off of Japanese Akoya oysters from infectious diseases. Furthermore, in 2011, tidal waves caused by the Great East Japan Earthquake of March 11 resulted in red tides that devastated the Akoya population. The pearl market has been plagued by small-scale die-offs and poor quality every year, and deteriorating quality has been linked to sudden changes in water temperature and phytoplankton management [14]. Therefore, aquaculture workers commonly use services that provide remote seawater temperatures in real time. However, these services provide seawater temperature measurements for at most two locations in one bay; thus, the seawater temperature is often different from the farm location's temperature. In addition, depending on the structure of the bay, sunshine and weather conditions result in differing temperatures, making temperature prediction difficult with the conventional global model. In this paper, we propose an algorithm that predicts seawater temperature in a marine aquaculture field by combining sea temperature data and actual weather data.

The second section of this paper demonstrates the importance of water temperature management in pearl farming and the problems to be resolved by this research. In Section 3, we describe our proposed prediction algorithm. The results of our evaluation experiments are set forth in Section 4, Section 5 introduces the previous research related to this research, and, finally, we summarize this paper in Section 6 and describe our future work. 


\section{Issues and current status of pearl farming}

\subsection{History and present situation}

Pearls have been prized all over the world since ancient times and, according to Yamada [12], used in jewelry since early BC. Prior to the establishment of pearl culture technology, only a few pearls were produced in 10,000 natural shells, and thus were highly valued due to their scarcity. In 1893 (Meiji 26), Aki Bay in Mie Prefecture was the first in the world to artificially cultivate pearls and, in the 1920s, the development of artificial cultured pearls stablized and they began to be supplied to the world. In the 1950s, Japan's share in the pearl market worldwide reached $90 \%$, playing a large part in Japan's export industry, with pearls coming from Mie, Wakayama, and Nagasaki Prefectures. However, pearl farming is now practiced around the world and Japan's share has decreased each year. In an effort to boost Japan's pearl farming, on June 7, 2017, the "Pearl Promotion Act" [12] went into effect. In addition, as a result of the losses of 1996, Mie prefecture, which has Japan's highest pearl farming output, purchased and interbred native Japanese Akoya oysters and non-Japanese pearl oysters in an effort to cultivate breeds that are resistant to environmental changes. Almost all cultivated pearl oysters are now crossbred species. However, the use of hybrids has resulted in the following problems:

- Fewer are evaluated as first grade (poor quality)

- As compared to a natural environment, aquaculture management is complicated (it is necessary to breed more than the required number)

- Hybrids suffer from contamination of egg cells (in the gonads) not found in Japanese giant pearl oysters (causes a deterioration in quality)

- Genetic information peculiar to Japan is lost (conservation of species)

We therefore promote rebuilding the brand image and sales of Japanese pearls by returning to pearl farming using Japanese Akoya oysters. However, Akoya oysters are weak against environmental change as compared with hybrids. Thus, the need for water temperature/water quality control to guard against red tides caused by massive zooplankton populations and death due to water temperature change is greater than for hybrids.

\subsection{Importance of present temperature control}

There are numerous processes for culturing pearls. In artificial culturing, a nacreous layer is formed by using a scalpel to insert a resin sphere (nucleus), which becomes the core of the pearl, into the oyster's body [12]. It takes three years and six months for the pearl to develop, so long-term management is required as compared with general fish farming. To insert the nucleus into a pearl oyster, it is intentionally stressed to weaken it by exposing the target pearl oysters to low-water temperatures. In addition, the proper water temperature for Akoya oysters is $10^{\circ} \mathrm{C}$ or more in winter and $25^{\circ} \mathrm{C}$ or less in summer. When wintering is over and a sharp rise in water temperature in summer is expected, it is necessary to lower the aquaculture to a place with a low water temperature. Seawater temperature is predicted by the experience and intuition of aquaculture workers and takes time to master. Therefore, the aging and retirement of aquaculture workers with experience and intuition is a threat to pearl farming's sustainability.

Seawater temperature collection and prediction are widely performed, but seawater temperature measurement using satellites for remote sensing can obtain only temperatures a few millimeters below the surface [7]. There is also a service [1] that predicts seawater temperature on a global scale using a model that integrates ocean currents and meteorological data. However, both of these methods target only seawater temperatures at the surface, whereas water temperatures at a depth between 2 to 10 meters is important for seafood cultivation for both fish and shellfish. For the farming of shellfish, such as pearls, scallops, and oysters, the shellfish are kept in a net, in seawater used for 
farming iced squid. etc. In order to install the net vertically, it is necessary to collect and predict each water temperature between 2-10 m.

The size of the area is also problematic for predicting temperature. For seawater temperature measurement and prediction using satellite images, a relatively wide measurement range of $1-\mathrm{km}$ mesh to $5-\mathrm{km}$ mesh is generally required. Seawater temperature also depends on surrounding geographical features, such as nearby marine farming. It is not uncommon for a rias-type terrain, as in Mie Prefecture's Ago Bay, which is the focus of this paper, to have differences of a maximum of $2{ }^{\circ} \mathrm{C}$ in the spring and $5{ }^{\circ} \mathrm{C}$ in the summer, depending on the surrounding geographical conditions. For sites used to farm fish, it is necessary to collect and predict temperatures in the range of $100 \mathrm{~m}$ - 500 m square. The Mie Prefecture Pearl Culture Council's "Ago Bay, Matoya Bay, Gokasho Bay Environment Monitoring System" [16] has been measuring the water temperature at depths of $0.5 \mathrm{~m}$, $2 \mathrm{~m}, 5 \mathrm{~m}$, and $8 \mathrm{~m}$ since 2007 and provides the data to aquaculture companies. The collected data includes examples of water temperatures in the summer and winter. The data for March 12, 2016 at 18:00 (spring season) are shown in Table 1 and summer data from August 20, 2016 at 18:00 are shown in Table 2. As mentioned earlier, in Julian Bay, which is characterized by a ria coastline, the seawater temperature change in each area is largely due to the situation in the surrounding areas. In particular, in the summer season, the bay front spot located in the inner part of Ago Bay is a tidal pool with a water temperature of $25^{\circ} \mathrm{C}$ or more, which adversely affects pearl oysters at a water depth of $0.5 \mathrm{~m}$ and $2 \mathrm{~m}$.

Table 1. Sea water temperature for 03/12/2016 18:00

\begin{tabular}{lllll} 
Name of Bay & $0.5 \mathrm{~m}$ & $2 \mathrm{~m}$ & $5 \mathrm{~m}$ & $8 \mathrm{~m}$ \\
\hline \hline Gokasho & 13.58 & 13.60 & 13.42 & 13.16 \\
Matoya & 11.53 & 11.49 & 11.35 & 11.42 \\
Inner of Ago & 13.04 & 13.11 & 12.84 & 12.72 \\
Center of Ago & 12.03 & 12.88 & 11.37 & 11.29
\end{tabular}

Table 2. Sea water temperature of 08/20/2016 18:00

\begin{tabular}{lllll} 
Name of Bay & $0.5 \mathrm{~m}$ & $2 \mathrm{~m}$ & $5 \mathrm{~m}$ & $8 \mathrm{~m}$ \\
\hline \hline Gokasho & 27.56 & 24.62 & 22.72 & 22.55 \\
Matoya & 25.23 & 23.11 & 22.85 & 22.12 \\
Inner of Ago & 28.11 & 27.81 & 25.04 & 24.62 \\
Center of Ago & 26.11 & 25.48 & 23.14 & 23.19
\end{tabular}

\begin{abstract}
As described above, in aquaculture, data collection and prediction in a fine measurement range are necessary. For that purpose, we propose highly accurate prediction by combining not only the water temperatures at each point, but also weather data.
\end{abstract}

\title{
3. Seawater temperature prediction using actual data
}

\subsection{Water temperature data and weather data collection device}

As mentioned in Section 2.2, since 2007, Mie Prefecture's Ago Bay has been using a device to measure water temperature. The existing equipment measures chlorophyll a concentration, salinity concentration, dissolved oxygen, and turbidity in addition to water temperature [? ]. However, due to the equipment's age and problems with the measuring equipment for measurements other than water temperature, it has been used only for water temperature since September 2007. The main reason is that accurate chlorophyll a and salt concentration measurement in seawater requires regular calibration of the sensors, resulting in high maintenance costs. Therefore, in response to the demand 
from pearl farmers, we introduced a device that can stably measure water temperature, which is the most important parameter for pearl culture. The old observation device of 2007 to 2017 and the new observation device currently in operation since 2017 are shown in Figure 1. As can be seen from the

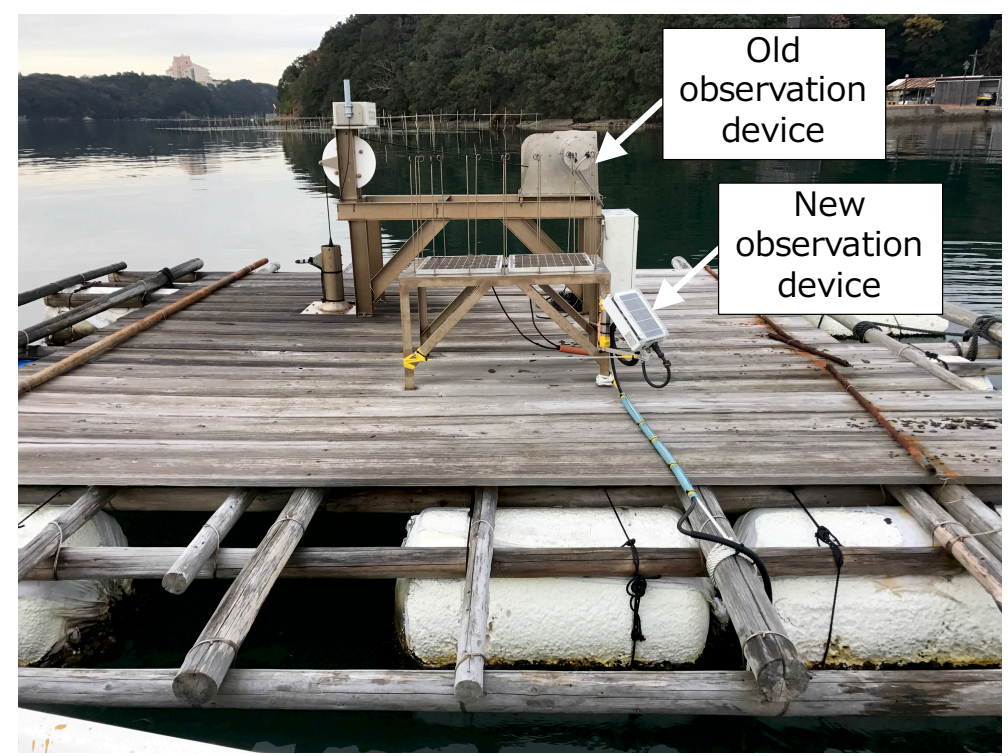

Figure 1. Old and new observation devices

exterior, the old observation device is large because it is necessary to lower the sensor to the set water depth. Both use a mobile phone network communication system and periodically send measurement data to the server. In this research, we propose an algorithm to predict seawater temperature for each depth at each point by using water temperature data from 2007 to 2017 collected by the old observation equipment.

\subsection{Seawater temperature prediction algorithm}

In this section, we describe the algorithm used for seawater temperature prediction. We use random forest as our prediction algorithm. Random forest is a machine learning method that can efficiently learn decision trees created in large quantities by utilizing the advantages of randomness. Especially when compared with the representative supervised learning Support Vector Machine (SVM), random forest gives more importance to feature quantity that can be calculated by learning, resulting in less overlearning [4]. Since random forest uses weak learning by decision tree, the decision tree learning is completely independent, and parallel processing is possible, learning can be performed at high speeds. In addition, because multiple models are generated by collective learning and the results of each model are integrated and combined to improve accuracy, it is suitable for a prediction environment where many parameters exist. The prediction of seawater temperature in this study uses four seawater temperatures for each point and many parameters, such as weather data and tidal current data. We obtained water temperature data for the past 10 years from the Mie Prefecture Pearl Farming Liaison Council's Ago Bay monitoring system. In addition, we collected data from the Ise Bay meteorological observatory nearest to Ago Bay, which is the observation point for 2007 to 2017 [17] from the database provided by the meteorological agency. Based on the above, multiple regression analysis by random forest was carried out at each of the following locations, and the prediction model was constructed and tested.

The following shows the location of the data acquisition and features of each location.

Gokasho Because it sits in the back of the bay, the effect of climate change is great, but there is not much influence from tidal changes. 
Matoya It touches Ise Bay's outdoor sea with a large temperature difference in water depths of $2 \mathrm{~m}$ and $8 \mathrm{~m}$.

Inner of Ago The temperature change of the layers is great (due to weather conditions) and the deviation of each water temperature is high with each tide.

Center of Ago Water temperature change is gentle.

South Ise meteorological observatory (weather data collection site)

An example of changes in seawater temperature due to Matoya Bay's weather conditions is shown in Fig. 2. Matoya bay is located in the vicinity of Ise Bay's outer sea, where seawater


Figure 2. Seawater temperature change and climatic conditions in Matoya Bay

temperature changes occur relatively slowly. However, due to a sudden temperature fluctuation on November 24 due to heavy rain, the surface layer had a drastic change in temperature with the middle layer also declining. As mentioned above, although seawater temperature varies with depth, weather also has a great influence. In this study, we model seawater temperature change at each point by using temperature and wind speed data as a weather condition, and construct a water temperature prediction algorithm.

\subsection{Data prediction flow}

We constructed our prediction model based on the actual data of each site. Random forest was used for the prediction algorithm and modeling. All calculations were done on Python and direct access to the database on the server was possible. We constructed this prediction model to use large-scale data. After modeling for each point, we input each day's weather forecast after its announcement by the Japan Meteorological Agency (JMA) at 15:00. In other words, by inputting the forecast values of hourly temperatures and wind speeds from the meteorological forecast data provided by the JMA, it is possible to predict seawater temperatures at depths of $0.5 \mathrm{~m}, 2 \mathrm{~m}, 5 \mathrm{~m}$, and $8 \mathrm{~m}$ at each site in one hour units. The data prediction flow is shown in Fig. 3.

We will describe the prediction results of the actual prediction model in the next chapter. 


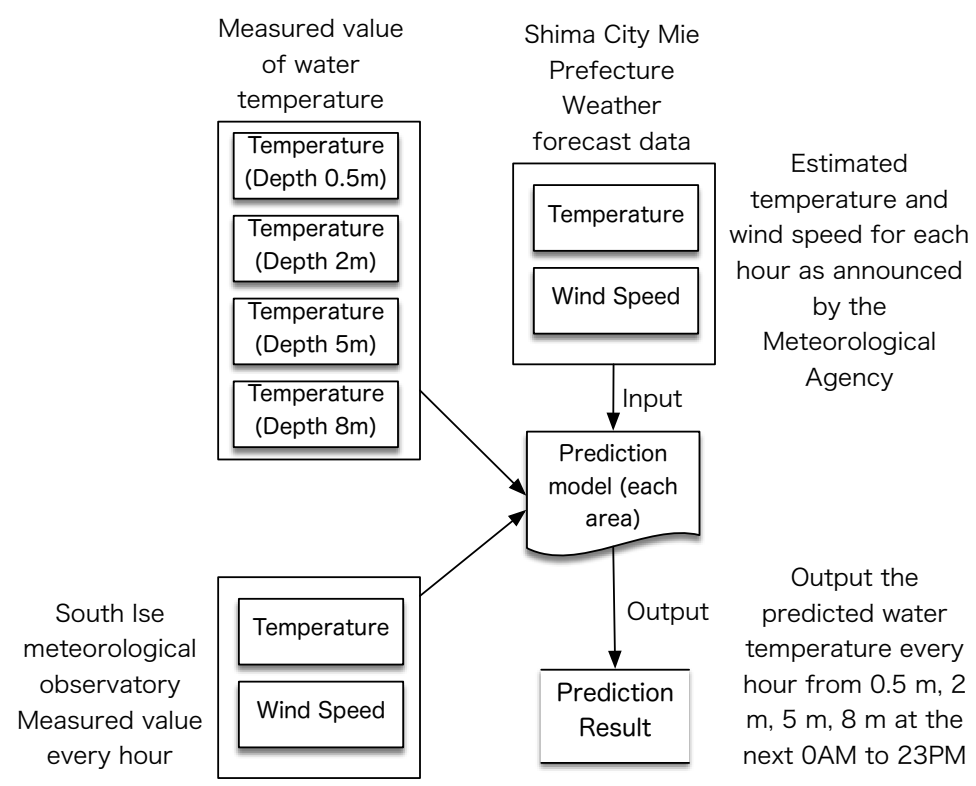

Figure 3. Data prediction flow

\section{Experimental result}

\subsection{Experimental setting}

In the evaluation experiments, we modeled using the water temperature data of each actual site as the learning data and meteorological data (hourly temperature and wind speed) every hour obtained from the South Ise meteorological observatory. Prediction accuracy was verified using the predicted values of temperature and wind speed per hour that are announced daily at 15:00 in Ise city, Mie prefecture. The data count for each data collection period and water temperature data is shown below.

\section{Data string used for model construction}

- Meteorological data measured value of South Ise meteorological observation (temperature, wind speed per hour) 03/20/2007 to 01/05/2016

- Actual measurement value of water temperature in Gokasho Bay (every hour) 03/20/2007 to 01/05/2016 - 72,923 cases

- Actual water temperature value of Matoya Bay (every hour) 03/20/2007 to 01/05/2016 - 76,254 cases

- Actual water temperature measurement for inner front of Ago Bay (every hour) 03/20/2007 to 01/05/2016 - 114,321 cases

- Actual water temperature measurement for center of Ago Bay (every hour) 03/20/2007 to 01/05/2016 - 96,957 cases

Maintenance and removal of land data due to typhoons was performed, so there were differences in the number of data. We describe our preliminary verification for verifying depth, model size, and prediction accuracy of the decision tree in the random forest method in the next section.

\subsection{Prior verification}

In this section, we examine the influence of depth of decision tree on prediction accuracy in the random forest method. With random forest, the depth of the tree structure in the decision tree is a parameter for adjusting the complexity of the model, and those with a deep decision tree structure are 
more complicated, while those with a shallow decision tree are simpler. To verify the maximum depth of the decision tree in this preliminary verification, we use Python's library scikit-learn to calculate the correct answer rate for each maximum depth of the decision tree, and use the 1.00 method to calculate the generalization performance of the model. The data used for this preliminary verification are:

- Meteorological measurement data of South Ise meteorological observatory (temperature, wind speed per hour) 03/20/2007 to 01/05/2016

- Actual water temperature measurement at a water depth of $8 \mathrm{~m}$ in Gokasho Bay (every hour) $03 / 20 / 2007$ to $01 / 05 / 2016-72,923$ cases

Next, the maximum depth of the decision tree was set to 40 and the generalization performance for each maximum depth was output. The correct answer rate for each maximum depth is shown in Table 3.

Table 3. Maximum depth of decision tree and accuracy rate

\begin{tabular}{|l|c|}
\hline Max depth & Correct answer rate \\
\hline \hline 5 & 0.325333 \\
\hline 10 & 0.326667 \\
\hline 15 & 0.326667 \\
\hline 20 & 0.325333 \\
\hline 25 & 0.94 \\
\hline 30 & 0.946667 \\
\hline 35 & 0.953333 \\
\hline 40 & 0.946667 \\
\hline
\end{tabular}

The results indicate that if the maximum depth of the decision tree is 25 or more, the generalization performance is approximately 0.94 . In the next section, we will discuss our verification of the prediction accuracy.

\subsection{Experimental result}

In this section, we describe the prediction results using actual data. For the prediction accuracy verification, we used the forecast data released by the JMA in Ise city, Mie prefecture as the input to the prediction model for each site using the data listed in Section 4.1. The forecast data released by the JMA is announced at 15:00 every day, and includes the forecast value of temperature and wind speed at every hour from 0:00 to 23:00 on the following day. By inputting the forecast data into the prediction model of each location, we obtain the forecast data of water temperatures at depths of 0.5 $\mathrm{m}, 2 \mathrm{~m}, 5 \mathrm{~m}$, and $8 \mathrm{~m}$ every hour from 0:00 to 23:00 on the following day, and compare it with the actual measured value of the water temperature at each depth of each site during the same period. The missing data in each data string is complemented by generating an intermediate value of the data before and after the time series. The maximum depth of the random forest decision tree in this accuracy verification is set to 30 based on the findings obtained in the preliminary verification in the previous section. The data sequence used for the prediction accuracy verification is shown below.

\section{Data string used for prediction accuracy verification}

- Meteorological measured data of South Ise meteorological observatory (temperature, wind speed per hour) 01/05/2016 to 01/05/2017 - 8,688 cases

- Actual water temperature measurement in Gokasho Bay (every hour) - 8,751 cases

- Actual water temperature measurement in Matoya Bay (every hour) - 8,748 cases

- Actual water temperature measurement in the inner front of Ago Bay (every hour) - 8,751 cases

- Actual water temperature measurement for center of Ago Bay (every hour) - 8,710 cases

In order to show which parameters are most effective for prediction, we performed prediction in two steps. Prediction 1 was made using only the water temperature and weather data, and prediction 
2 was made by learning using the temperature and wind speed for each water temperature and meteorological data. The prediction 1 result is shown in Table 4 and prediction 2 result is shown in Table 5. Regarding error in the prediction result, the predicted value of the water temperature at each water depth/point for January 6, 2016 to January 7, 2017 output by the prediction model is compared with the actual measured value at each point. Differences after comparison are averaged. The one with the largest error when comparing is the maximum error.

Numerical values after the comma at each water temperature indicate the maximum error at each depth. The maximum error at each point is also shown in the table.

Table 4. Result 1: Temperature only

\begin{tabular}{|l|c|c|c|c|c|}
\hline Area & $0.5 \mathrm{~m}$ & $2 \mathrm{~m}$ & $5 \mathrm{~m}$ & $8 \mathrm{~m}$ & Maximum error \\
\hline \hline Gokasho & $1.175,7.10$ & $1.113,6.46$ & $1.083,6.25$ & $1.095,6.14$ & 6.46 \\
\hline Matoya & $1.157,6.10$ & $1.171,5.68$ & $1.158,6.62$ & $1.141,7.37$ & 7.37 \\
\hline Inner of Ago & $1.121,11.9$ & $1.188,11.6$ & $1.136,12.24$ & $1.079,10.47$ & 12.24 \\
\hline Center of Ago & $1.157,6.39$ & $1.109,7.01$ & $1.070,6.25$ & $0.969,5.68$ & 7.01 \\
\hline
\end{tabular}

Table 5. Result 2: Temperature and wind speed

\begin{tabular}{|l|c|c|c|c|c|}
\hline Area & $0.5 \mathrm{~m}$ & $2 \mathrm{~m}$ & $5 \mathrm{~m}$ & $8 \mathrm{~m}$ & Maximum error \\
\hline \hline Gokasho & $1.008,5.99$ & $0.978,6.06$ & $0.951,5.46$ & $0.971,6.49$ & 6.49 \\
\hline Matoya & $1.029,5.60$ & $1.051,5.76$ & $1.042,5.80$ & $1.030,6.16$ & 6.16 \\
\hline Inner of Ago & $1.042,12.9$ & $1.060,11.6$ & $1.006,11.76$ & $1.030,6.16$ & 12.91 \\
\hline Center of Ago & $0.994,6.56$ & $0.971,6.52$ & $0.938,6.32$ & $0.853,5.57$ & 6.57 \\
\hline
\end{tabular}

\subsection{Discussion}

It was also found that the model's mean error based on the combination of air temperature and seawater temperature in prediction result 1 is about $1.1^{\circ} \mathrm{C}$. Further, as for the result of the prediction model with the wind speed added in prediction result 2 , since the average error is approximately 1 ${ }^{\circ} \mathrm{C}$, it is possible to predict in the normal state. However, the maximum error exceeds $6{ }^{\circ} \mathrm{C}$ regardless of either result, and the maximum error is $12.9{ }^{\circ} \mathrm{C}$ for inner Ago bay. The cause of this error in the prediction result is a sudden temperature change. In particular, with regard to the early winter seasons, the temperature fluctuation range was large and deviation between the predicted result and the measured value occurred with temperature change. A graph of the predicted value and measured value of December 26-27, 2016, in which the error between the predicted value and the measured value was at its maximum, is shown in Fig. 4.

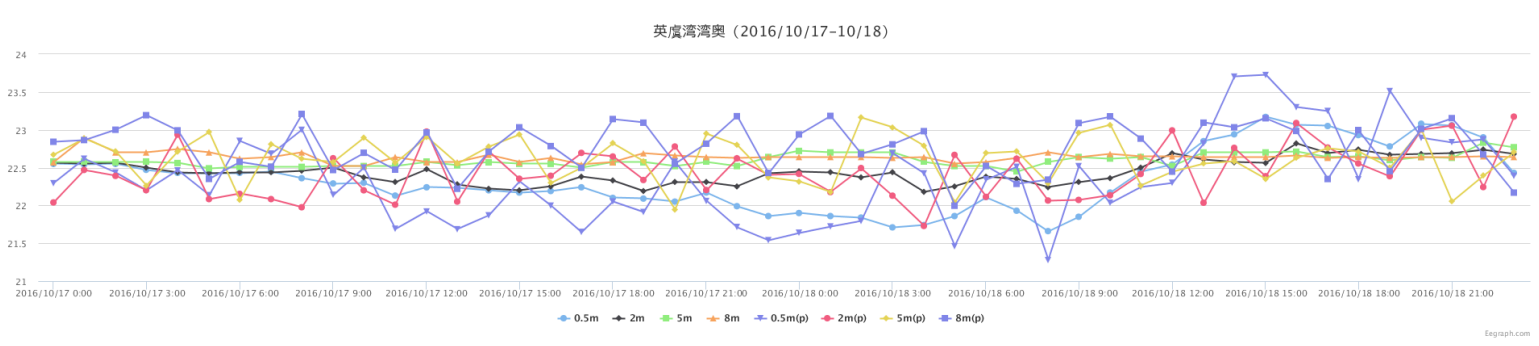

Figure 4. Predicted value and measured value of 12/26-27/2016

On December 26, 2016, the high pressure that had prevailed up until December 25, 2016 suddenly collapsed due to the passage of low pressure accompanied by a front. Sudden changes in weather with little accompanying data increase the maximum error; thus, performance cannot be improved with respect to the data at normal times, which is often contained in the training data, and 
overlearning occurs. We believe that prediction accuracy can be improved by continuing to alter the algorithm, applying measures to prevent excessive learning, and adding parameters such as tidal currents, wind speed, and total rainfall.

In this research, we made predictions by using weather forecast data (hourly temperature and wind speed forecast) provided by the JMA. The JMA's weather forecast for the following day is predominantly $87 \%$ accurate in terms of the forecast for rain and roughly $85 \%$ in terms of forecast error of the highest temperature [6]. Thus, we believe this information to be accurate for input as a forecast of seawater temperature. However, rainfall amounts may differ between the water temperature observation point and the meteorological observation point of the South Ise meteorological observatory because they are two separate locations. We plan to use tidal data provided by the JMA. In the future, we aim to confirm the minimum data required for learning, cope with outliers, and predict long-term seawater temperature using long-term forecast data. For the inner Ago bay area, the meteorological data acquisition point is far and is surrounded by mountains. Therefore, using our wireless sensor network (WSN) platform, in April 2017, we installed a composite weather meter on the same raft as the water temperature observation device. This complex meteorological meter is shown in Fig. 5.



Figure 5. Meteorological sensor at actual field

This method of continuously collecting this weather meter's data and combining it with weather data in the vicinity of the site where we want to predict seawater temperature reduces errors. 


\section{Related Works}

\subsection{Research on remote sensing}

Modeling and forecasting of tidal currents and seawater temperature have long been conducted in the field of oceanography [18]. Recently, in addition to aircraft, marine environmental information is obtained and predicted based on various sensors mounted on artificial satellites. For example, research on ocean environment prediction uses satellite images, land and ocean weather conditions, and sea temperature data obtained by sensing buoys [1] Research to predict atmospheric and oceanic conditions using a relatively small range of 2-20-km mesh [3] has improved the sensing accuracy of sea surface temperature using infrared and microwave sensors mounted on artificial satellites [7]. Studies have investigated the relation between catch size and seawater temperature in an attempt to quantify the relationship between seawater temperature change and fishery [8], and used satellite images to determine the growth of coral reefs [9]. However, these studies do not provide the seawater temperature of farmed sealife, which is most important for fishery and marine aquaculture. The sea surface temperature is easily influenced by weather conditions such as surrounding air temperature. Compared to the surface temperature of the ocean, seawater temperature changes tend to stabilize as the water depth increases, which is different from the sea surface temperature, which changes largely depending on temperature change. Particularly in the summer and winter, the sea surface temperature is often approximated to the atmospheric temperature due to the influence of solar radiation and temperature change, but these factors have a lesser impact on middle sea water temperature. Therefore, it is important to forecast not only the sea surface temperature via remote sensing, but also the seawater temperature of the water depth actually used for farming.

\subsection{Research on environment information gathering and prediction by WSN}

WSN technology forms the core of IoT (Internet of Things) and M2M (Machine to Machine) and has been extensively studied [19]. The nodes constituting the WSN can constitute a "multi-hop/ad hoc network" that acquires sensor data, such as temperature, illuminance, acceleration, and the like, and transfers the acquired data by a bucket brigade method using radio waves [20],[21]. WSN reduces autonomous network construction by simply arranging nodes, so it can reduce the installation work at the site. When acquiring sensor data, because we can capture the dynamics of the world, WSN is widely studied as a promising application for object tracking and monitoring of the natural environment.

We are developing sensor network devices and server applications capable of gathering high-density information on a large scale and collecting environmental data [22].

\section{Conclusion}

In this paper, we proposed an algorithm to predict seawater temperature at water depths used for aquaculture. Sucn prediction for the water depth actually used for farming as proposed in this research has heretofore not been carried out, despite its importance in successful aquaculture. We proposed an algorithm using a prediction model based on actual weather data and seawater temperature data that has a high prediction accuracy of about $1{ }^{\circ} \mathrm{C}$. We will continue our research on reducing outliers, coping with overlearning, and long-term seawater temperature prediction on a monthly basis. In the future, we will support not only seawater temperature, but also chlorophyll a and salinity concentrations to further promote sustainable aquaculture.

\section{References}

1. Chassignet, Eric P., et al., "Ocean prediction with the hybrid coordinate ocean model (HYCOM)." Ocean Weather Forecasting, Springer Netherlands (2006): 413-426. 
2. Shriver, Jay F., et al. "1/32 ${ }^{\circ}$ real-time global ocean prediction and value-added over $1 / 16^{\circ}$ resolution." Journal of Marine Systems 65.1 (2007): 3-26. ,

3. Hodur, Richard M., "The Naval Research Laboratory' s coupled ocean/atmosphere mesoscale prediction system (COAMPS)." Monthly Weather Review 125.7 (1997): 1414-1430.

4. $\quad$ Breiman, Leo, "Random forests." Machine learning 45.1 (2007): 5-32.

5. tenki.jp, Weather Data (12/26/2016) http://www.tenki.jp/past/2016/12/26/

6. Japan Meterological Agency, "Result of accuracy verification of weather forecast." http://www.data.jma.go.jp/fcd/yoho/kensho/yohohyoka_top.html

7. Lobitz, Brad, et al., "Climate and infectious disease: use of remote sensing for detection of Vibrio cholerae by indirect measurement." Proceedings of the National Academy of Sciences 97.4 (2000): 1438-1443.

8. Cox, Charles, "Some problems in optical oceanography." J. Mar. Res. 14 (1955): 63-78.

9. Mumby, Peter J., et al., "Remote sensing of coral reefs and their physical environment." Marine Pollution Bulletin 48.3 (2004): 219-228.

10. Hosoda, Kohtaro, Kawamura, Hiroshi, Lan, Kuo-Wei, Shimada, Teruhisa, and Sakaida, Futoki, "Temporal scale of sea surface temperature fronts revealed by microwave observations." IEEE Geosci. Rem.Sens. 9 (2011).

11. Tategami area pearl farming reconstruction project, NPO corporation fishery industry, fishing village activation promotion organization, http:/ /www.fpo.jf-net.ne.jp/, 04/02/3027

12. Yamada, Atsumi, World History of Pearls, ISBN 978-4-12-102229-5

13. Act on promotion of pearl (Act No. 74 of June 7, 2016), Japan Law database, http:/ /law.e-gov.go.jp/htmldata/H28/H28HO074.html

14. Higuchi, Keita, Nagai, Kiyohito, Hattori, Fumihiro, et al., "Compost from pearl culture waste: method of composting and effective use." Nippon Suisan Gakkaishi, Vol.82, No.4 (2016): 608.

15. Fishing season situation, Mie Prefectural Fisheries Research Institute, http://www.pref.mie.lg.jp/suigi/hp/000200511.htm

16. Mie Prefecture pearl farming related fishing ground water temperature monitoring system, Mie Prefecture Pearl Farming Liaison Council, http:/ / www.ohyamanet.info/ m-shinkyo/index.php

17. Japan Meteorological Agency, Past weather data search, http://www.data.jma.go.jp/obd/stats/etrn/index.html

18. Kumar, M. and Monteith, J.L., "Remote sensing of crop growth." Plants and the Daylight Spectrum (1981): 133-144.

19. Akyildiz, I. F., Su, W., Sankarasubramaniam, Y., and Cayirci, E., "Wireless sensor networks: a survey," Computer Networks, Vol. 38 (2002): 393-422, Elsevier Science B. V.

20. Chong, Chee-Yee and Kumar, Srikanta P., "Sensor networks: evolution, opportunities, and challenges," Proceedings of the IEEE 91.8 (2003): 1247-1256.

21. Kuorilehto, Mauri, Hannikainen, Marko, and Hamalainen, Timo D., "A survey of application distribution in wireless sensor networks," EURASIP J. Wirel. Commun. Netw., 5, 5 (2005): 774-788.

22. Otsuka, Takanobu, Torii, Yoshitaka, and Ito, Takayuki. "Anomaly weather information detection using wireless pressure-sensor grid,” Journal of Information Processing (JIP), Vol.23, No.6, Information Processing Society of Japan (IPSJ) (2015). 